\title{
Corrigendum: Histone deacetylase inhibitors reverse gene silencing in Friedreich's ataxia
}

David Herman, Kai Jenssen, Ryan Burnett, Elisabetta Soragni, Susan L Perlman \& Joel M Gottesfeld

Nature Chemical Biology 2, 551-558 (2006); published online 20 August 2006; corrected after print 22 May 2007

In the version of this article initially published, no competing financial interests were declared. The authors now declare that they have competing interests that might be perceived to influence the results and discussion reported in this paper. The error has been corrected in the HTML and PDF versions of the article. 